\title{
Modeling and Optimization for Maintenance Support Human Unit based on Multi-objective Constraint
}

\author{
Qiao-ran Ran ${ }^{\mathrm{a}}$, Qiang Wang ${ }^{\mathrm{b}}$,Zheng Sun ${ }^{\mathrm{c}}$ and Sheng Pang \\ Army Engineering University, Shijiazhuang, China

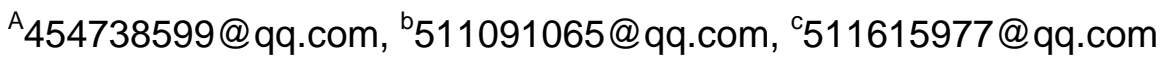

\begin{abstract}
Aiming at the personnel optimization problem exist in equipment maintenance support unit, the personnel optimization problem of support unit is studied in this paper. Firstly, the relation between the maintenance time and personnel quantity is analyzed, the multi-objective model of maintenance time and maintenance person is build. Secondly the models and the corresponding project are studied based on the theory of modified ant colony algorithm. Finally, the validity and practicality of the optimization model and solving method are verified through example, and the feasibility of conclusion is externally analyzed. The research provides the basis for personnel optimization of maintenance support unit, and it features high engineering application value for the equipment maintenance work of all levels repair organizations.
\end{abstract}

Keywords: Optimization; maintenance support human unit; personnel optimization; ant colony algorithm.

\section{Introduction}

The equipment maintenance support human unit is an effective unit to carry out the maintenance task and is the basic unit of the maintenance resources. The equipment maintenance activities are usually thought the basic unit of maintenance teams or groups. These maintenance teams or groups are the most basic unit of composed maintenance manpower and completed maintenance tasks [1]. In the determination of the human unit number at present, mainly according to the actual work of a large number of historical datas, and using the application of empirical reasoning method to determine. The number of maintenance personnel in the presence of fuzzy matching, reasonable and scientific difference. Moreover, because of the replacement of the equipment, the improvement of the maintenance tools and equipment, and the improvement of the technical ability of the personnel, which leads to the further increase of the number of maintenance personnel and the lack of the support of quantitative method. So how to quantitatively determine support units number, optimize maintenance power structure, build a reasonable unit maintenance power, can assure to minimal staff in the shortest possible time to complete maintenance tasks is a practical problem to be solved at present. There is an urgent need for a set of scientific and reasonable basic maintenance unit allocation method for maintenance of the power unit of allocation to provide technical support. In this context, the security forces unit repair allocation problem and solution research.

\section{Optimization Model of Maintenance Support Human Unit}

\subsection{Problem description}

Equipment maintenance work usually involves a number of complex work processes, these processes are completed by a number of types of work. To co-ordinate arrangements for repair work, usually will repair tasks according to type of work is decomposed into a number of work, each work includes a number of processes and process node repair time is different. So, the entire project period of repairing is by the process of node of time to repair decision, to optimize the number of maintenance personnel, it is necessary to process node repair time are discussed. There is a certain relationship between the repair time and the number of the process nodes, which is the key to optimize the maintenance personnel. 
Under ideal conditions, equipment and facilities is perfect, spare parts supply sufficient, the maintenance period of process node is fixed, the procedure node repair time will shorten with the increase in number of repair part in, without considering the difference in the personnel to repair ability. When the number of repairs is fixed, the actual working hours of each process are also fixed. But it is not possible to increase the time limit for the duration of the staff. When the number of people increases to a fixed value, the increase of the number of people becomes small, and the variation of the time is in a certain range [2]. As shown in Figure.1.

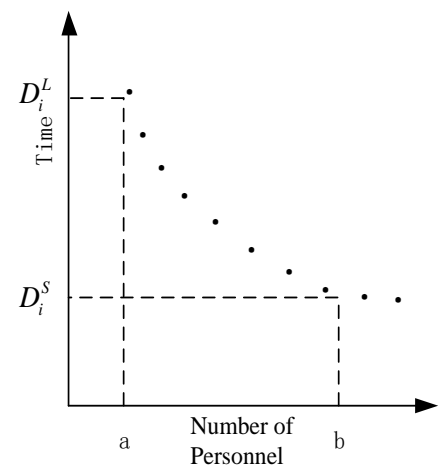

Fig. 1 The discrete relationship between the time and maintenance personnel quantity

Since the value of the number of maintenance personnel is an integer, the relationship between time and personnel is represented as a number of discrete points in the graph. In Figure 1, the minimum number of repair work required to complete a repair process is $a$, the corresponding repair time is $D_{i}^{L}$. With the increase of the number, the repair time is gradually reduced, but the process of repair time is not possible with the increase in the number of personnel to a fixed value, the impact of time becomes very small, the process of the limit time is $D_{i}^{s}$ in the graph, that is, the number of repair process is $b$.

From the discrete function relationship between the repair time and people number, it can be considered that each repair procedure has multiple execution plans. Each one is composed of a fixed repair time and a fixed number of maintenance personnel. In order to minimize the total duration and total number of people at the same time, it is the problem of multi-objective optimization.

\subsection{Model construction}

In order to establish a multi-objective optimization model, the following assumptions are made.

(1) each repair process has a certain sequence of order, only after the completion of all the close before the process can begin, and repair work once you start, you will not be interrupted until the end.

(2) The repair time of each process has the maximum $D_{i}^{L}$ and minimum values $D_{i}^{S}$, and both in the maximum and the minimum range.

(3) The total duration of the whole repair work was calculated under the certain working hours of each operation. It also equals to the length of the key line in the process of maintenance.

The mathematical model can be expressed as:

$$
\begin{aligned}
\min H_{T}= & F\left(H_{1}\left(t_{1}\right), H_{2}\left(t_{2}\right), \cdots, H_{n}\left(t_{n}\right)\right) \\
& \min T_{N}
\end{aligned}
$$




$$
\begin{aligned}
& \text { st. } \\
& \left\{\begin{array}{l}
t_{j}-t_{i} \geq d_{j}, \quad i \in P(j) \\
H_{i}=\sum_{k=1}^{O P_{i}} \delta_{i k} H_{i k} \\
d_{i}=\sum_{k=1}^{O P_{i}} \delta_{i k} d_{i k} \\
\sum_{k=1}^{O P_{i}} \delta_{i k}=1, \quad \delta_{i k}=0 o r 1
\end{array}\right.
\end{aligned}
$$

Where: $t_{i}$ is the end time of the $i$ working procedure, $d_{i}$ is the repair time length of $i$ working procedure, $T_{N}$ is the actual duration of repair work, $O P_{i}$ is the repair plan number can be choose of $i$ working procedure, $H_{i k}$ is the number of repair personnel during $d_{i}=d_{i k}\left(k=1,2, \cdots, O P_{i}\right)$ time of $i$ working procedure, $\delta_{i k}$ is the switch variable, if the $\delta_{i k}=1$ means the $i$ working procedure use the $k$ maintenance program.

$P(j)=\{i \mid i$ is the pre-tightening procedures of $j\}$, where $P(j)$ is the pre-tightening procedures collection of $i$ working procedure.

The Formula (2) is the maintenance period constraint, which ensure that the repair time of the procedure is allowed. Formula (3) is the procedure constraint, which ensure that the maintenance sequence of the nodes is in accordance with the logical relationship. The remaining constraints ensure that the implementation of each process is feasible. The function $F$ of total maintenance number and number of each repair node in objective function can be calculated by standard network planning method. Through time personnel demand superposition method, determine the personnel demand in each period, the objective function value $H_{T}$ is the personnel quantity when the most personnel required $[3,4]$.

\subsection{Improvement of multi-objective model}

Each procedure of the repair project is a work of a node, and all procedures are arranged in a sequence of order to form a complete maintenance task, as shown in Figure. 2.

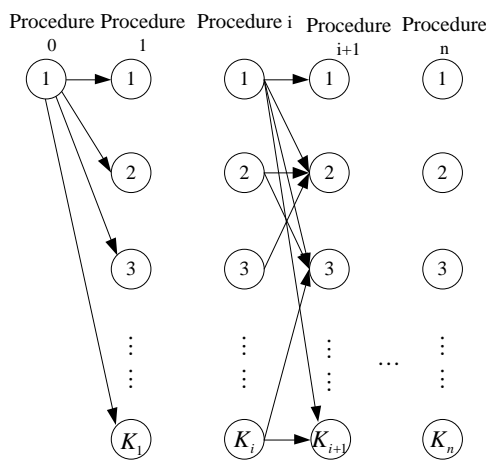

Fig. 2 Schematic diagram of path combination optimization problem

Each node in the graph represents the different maintenance plan of the corresponding procedure. Set 0 is a virtual procedure to represent the beginning of the project, the $j$ node of in the $i$ column indicates that the procedure $i$ using the $j$ maintenance programs. The edge of the graph represents the maintenance plan of two adjacent procedures, $\left(i, j_{1}, j_{2}\right)$ indicates that the procedure $i$ using the $j$ maintenance programs, and the procedure $i+1$ using the $j+1$ maintenance programs. Each of the paths from the 0 column to $n$ column indicates a full implementation plan for the repair project. The maintenance personnel optimization problem is actually found the optimal path from the 0 column to $n$ column in the graph, the time and maintenance personnel number is the shortest. 


\subsection{Transformation of the objective function}

The transformation of the objective function is transformed into a single objective by using the adaptive weight method, calculate the best comprehensive value of time and personnel project. The basic idea of the Adaptive Weight Method is to set the weight value of each objective function, and then turn to a single objective function, so as to tend to ideal point when search optimal solution. In this paper, the modified adaptive weight method, which is proposed by Daisy et al., is used to calculate the weight of the time and the people's objectives. Calculated as follows[5]:

(1) When $X_{r}^{\max } \neq X_{r}^{\min }$ and $X_{g}^{\max } \neq X_{g}^{\min }$,

$$
\begin{gathered}
\omega_{r}=\frac{\frac{X_{r}^{\min }}{X_{r}^{\max }-X_{r}^{\min }}}{\frac{X_{r}^{\min }}{X_{r}^{\max }-X_{r}^{\min }}+\frac{X_{g}^{\min }}{X_{g}^{\max }-X_{g}^{\min }}} \\
\omega_{g}=\frac{\frac{X_{g}^{\min }}{X_{g}^{\max }-X_{g}^{\min }}}{\frac{X_{r}^{\min }}{X_{r}^{\max }-X_{r}^{\min }}+\frac{X_{g}^{\min }}{X_{g}^{\max }-X_{g}^{\min }}}
\end{gathered}
$$

(2) When $X_{r}^{\max }=X_{r}^{\min }$ and $X_{g}^{\max }=X_{g}^{\min }$,

$$
\omega_{r}=\omega_{g}=0.5
$$

(3) When $X_{r}^{\max }=X_{r}^{\min }$ and $X_{g}^{\max } \neq X_{g}^{\min }$,

$$
\omega_{r}=0.9, \omega_{g}=0.1
$$

(4) When $X_{r}^{\max } \neq X_{r}^{\min }$ and $X_{g}^{\max }=X_{g}^{\min }$,

$$
\omega_{r}=0.1, \omega_{g}=0.9
$$

Where: $X_{g}^{\max }$ and $X_{g}^{\min }$ is indicated that the maximum and minimum values of maintenance time in all the solutions generated by the Ant Colony Algorithm in one cycle. $X_{r}^{\max }$ and $X_{r}^{\min }$ is indicated that the maximum and minimum values of maintenance personnel in all the solutions generated by the Ant Colony Algorithm in one cycle. $\omega_{g}$ is the adaptive weight value of maintenance time, $\omega_{r}$ is the adaptive weight value of maintenance personnel.

In the reference Genetic Algorithm, the method of the objective function is normalized, and the integrated target value of the time limit for a project is calculated according to the Formula (6):

$$
f(Y)=\omega_{g} \frac{X_{g}-X_{g}^{\min }+c}{X_{g}^{\max }-X_{g}^{\min }+c}+\omega_{r} \frac{X_{r}-X_{r}^{\min }+c}{X_{r}^{\max }-X_{r}^{\min }+c}
$$

Where: $Y$ is the serial number of the solution generated in the Ant Colony Algorithm, $X_{g}$ and $X_{r}$ is the target value of the maintenance time and maintenance personnel for the $Y$ solution. In order to avoid the comprehensive value is invalid or 0 , the random number $C$ obey the uniform distribution $[0,1]$ is introduced.

\section{Basic Ant Colony Algorithm for the Optimization Problem Solving}

\subsection{Basic principles of Ant Colony Algorithm}

In 1990s, a new stochastic search algorithm is proposed through simulating the behaviour of the natural ants foraging by Italy scholars Doirgc, et al. Each ant will leave a certain amount of pheromone in the process of looking for food, used to mark the path of their own back to the nest, and mark the food direction for other ants. When they encounter a road that has not passed, they randomly choose a path along the path, and release the pheromone about the length of the path. The longer the path of the ant, the smaller the amount of pheromone released. When other ants again encounter this intersection, they will choose the larger amount of pheromone on the road, so that the pheromone of a positive feedback mechanism. The amount of pheromone on the optimal path will become more and 
more large, while the pheromone on other paths will decrease with the increase of time, and finally the whole ant colony will find the optimal path. Summary of the behaviour of the ant colony can be extracted from the three mechanisms. Firstly, the foraging mechanism of the ant colony. Secondly, pheromone update mechanism on the path. Finally, the pheromone gathering mechanism [6].

In this paper, the Ant Colony Algorithm is used to solve the problem of maintenance support personnel optimization, the ant represents the support unit. In order to meet the requirements of important degree and time constraints, the optimal objective value is the most appropriate support unit personnel quantity among the support point. With the maintenance time of each point as the pheromone information, the probability of the formation of the mobile is chosen, short maintenance time with high probability are selected, long time choice probability is small, so the moving path of ants is determined. At the same time, according to the repair time of each node is determined pheromone, long maintenance time with lower pheromone, short maintenance time pheromone more on the contrary. Pheromone updates on the path is to consider the importance of demand point and time to determine the degree of urgency, the more important and urgent, the pheromone volatile more slowly, and more reserved.

\subsection{Path construction}

According to the basic principle of Ant Colony Algorithm, the key link of the algorithm is the feeding path formation and the pheromone updating. The path construction is combined with heuristic information to determine the probability that the ants are moving from the current point to the next point. The maximum probability points as the following points, the whole path is gradually forming. The probability of the movement of the ants is shown in Formula (7) [7] .

$$
P_{i j}^{k}(t)=\left\{\begin{array}{cc}
\frac{\left[\tau_{i j}^{k}(t)\right]^{a}\left[\eta_{i j}^{k}(t)\right]^{\beta}}{\sum\left[\tau_{i j}^{k}(t)\right]^{a}\left[\eta_{i u}^{k}(t)\right]^{\beta},} & j \in J_{n}(i) \\
0, & \text { others }
\end{array}\right.
$$

Where: $\tau_{i j}^{k}(t)$ is the concentration of pheromone on the path $J$ at time $t$, the concentration of pheromone on every path is equality at initial time, $\tau_{i j}^{k}(0)=C$. The $\eta_{i j}^{k}(t)$ is the heuristic information value which is related to the path $i$ in the research problem, $\eta_{i j}^{k}(t)=1 / c_{i j}$. The ant comprehensive considering the pheromone account on the path and the value of information in the moving process, then determines the direction of the transfer. The $\alpha$ and $\beta$ is the parameters of the weight in the probability, which is the control of pheromone and heuristic information value.

\subsection{Pheromone update}

Pheromone will be reduced with the passage of time, if follow another ant through the path, the path pheromone can be increased. The parameter $\rho$ means the pheromone evaporation coefficient [8]. In this study, the support personnel unit choose demand point according to the important degree and the latest start time. The important degree is high point where the path information prime less volatile, the latest start time is short of the security requirements pheromone trails is less volatile. The parameter $\rho$ is changed to the function, which is inversely correlated to the degree $q_{j}$, and proportional to the latest start time $T_{L}^{j}$. The ant number in the colony is expressed as $M$, the pheromone increased because many ants through the path is expressed as $\sum_{k=1}^{M} \Delta \tau_{i j}^{k}$. After a completed moving path, the pheromone update according to equation (8), all the paths are updated, the next ants are placed in the new round [9].

$$
\begin{gathered}
\tau_{i j}^{k}(t+1)=\left[1-\rho\left(q_{j}, T_{L}^{j}\right)\right] \cdot \tau_{i j}^{k}(t)+\sum_{k=1}^{M} \Delta \tau_{i j}^{k} \\
\Delta \tau_{i j}^{k}=\left\{\begin{array}{l}
Q / c_{i j}, \quad \text { The ant } k \text { pass } i j \\
0, \quad \text { The ant } \mathbb{k} \text { not pass } i j
\end{array}\right. \\
\rho\left(q_{j}, T_{L}^{j}\right)=3 \rho_{0} \cdot T_{L}^{j} /\left(q_{j} \cdot T\right)
\end{gathered}
$$


Where: $\tau_{(i j)}(t+1)$ and $\tau_{(i j)}(t)$ respectively express the intensity of each side of the edge after the $T$ cycles and the $T+1$ cycles. The $\rho$ is the constant between [0,1], express the volatile factor of the pheromone on the path.

\subsection{Solving basic process of Ant Colony Algorithm}

According to the principle of Ant Colony Algorithm, the process which personnel multi-objective optimization model determined can be divided into four parts: The first step, initialization, according to the actual situation to determine the parameters of the algorithm. The second step, determine the number of ants, grouping and signing each ant. The third step, randomly assigned support demand points for each ant, scheduled route is determined according to ant moving selection probability. The forth step, calculate and determine the optimal target value after all groups paths are determined. The path pheromone updated, to start the next cycle, the final optimal objective value and number of support teams is determined. The specific process steps are shown in Figure. 3[8][10].

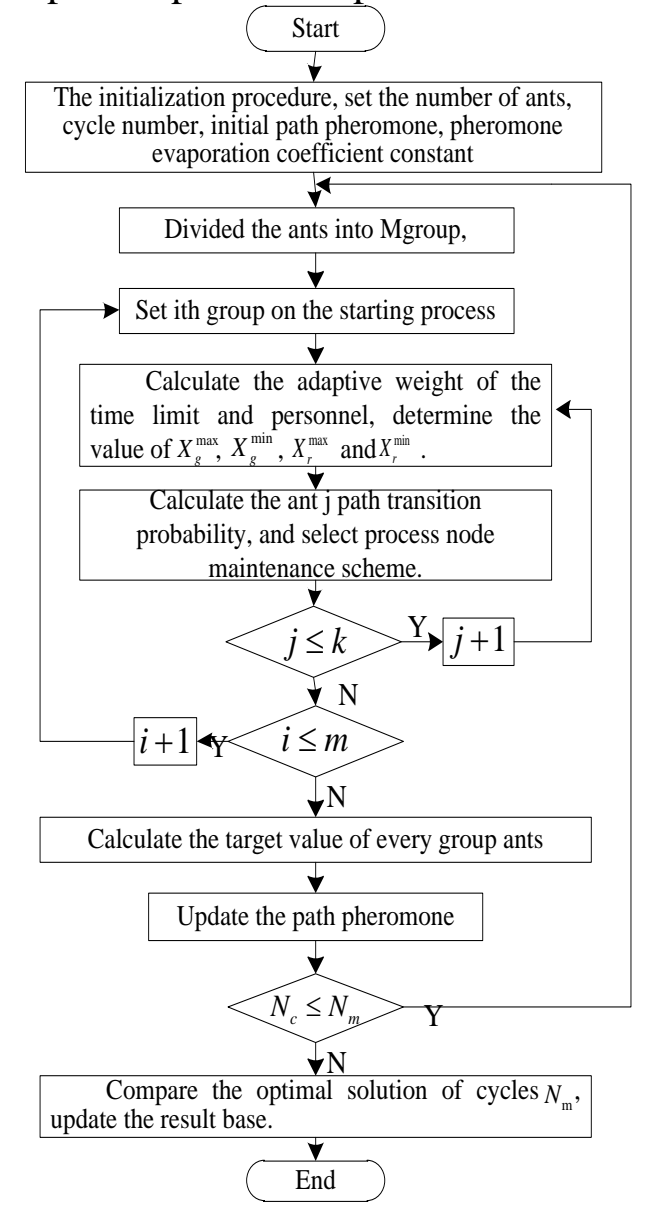

Fig.3 the algorithm process of the optimization model for time and personnel quantity

(1) Determine the maximum number of cycles $N_{\mathrm{m}}$ and the number of ants $M$, give the same initial value to pheromone of each side of the Figure 2, generate the initial solution by random method.

(2) Calculate the maintenance period and personnel use the standard network diagram method for the route which each ant searched out.

(3) Establish base solution, and then separate the solution of Pareto E. After the new solutions generated, if the current solution exists in solving library, then output duration and personnel value, if not, then calculate the time limit and the personnel, according to the definition of Pareto solution, delete the non optimal from the solution, forming Pareto solution $E$.

(4) Calculate the adaptive weight of the time limit and personnel, determine the value of $X_{g}^{\max }$, $X_{g}^{\min }, X_{r}^{\max }$ and $X_{r}^{\min }$.

(5) Calculate the comprehensive value of the time limit and the personnel's objectives. 
(6) The pheromone intensity of each side is updated by the formula (8) after one cycle.

(7) The ants choose the path according to the pheromone intensity on each side, and the probability of each side selection is obtained by the formula (7).

The $\alpha$ and $\beta$ are the parameters to adjust the pheromone and the path visibility. The $\left(\eta_{i, j_{1}, j_{2}}\right)$ is the edge visibility, which express the local information in the search process. The $\eta_{i, j_{1}, j_{2}}=1 / G_{i+1, j_{2}}, G_{i+1, j_{2}}$ is the time limit and personnel comprehensive value according to the adaptive weights when the process $i+1$ using the scheme $j_{2}$, the dimension does not considered, the heuristic information impact on the path selection is only express.

(8) if the current iteration number $\mathrm{N}$ is less than the number of cycles $N_{\mathrm{m}}$ of the set, the pheromone on each path is updated, the procedure turn to the (3) step, else turn to the (9) step.

(9) The new solution added to solutions of library, and update the Pareto solution set E same time, repeat the step (4) to the (8) until the number of iterations so far.

\section{Application}

\subsection{Case calculation}

With a certain type of ordnance equipment chassis repair cases, part of the repair work flow as shown in Fig.4, including in 25 procedures, each procedure has many kinds of solutions. In the process of personnel optimization of the maintenance work of this type of equipment, the optimization of the model is transformed into a combinatorial optimization problem by model pre-processing. In fact, it is the choice of the solution for each process, which is the minimum of the time limit and personnel through the combination of these schemes.

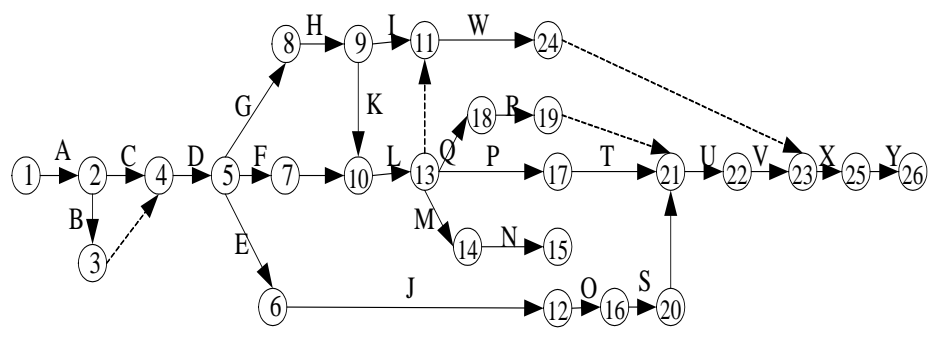

Fig.4 a part of ordnance equipment repair work process

The simulation data of executable maintenance plan of process and the corresponding repair time and personnel number of each maintenance plan are shown in Table 1.

Table 1 maintenance plan and personnel number simulation data

\begin{tabular}{ccccccccccc}
\hline \multirow{2}{*}{ Procedure } & \multicolumn{7}{c}{ Programme1 } & \multicolumn{7}{c}{ Programme2 } & \multicolumn{7}{c}{ Programme3 } & Programme4 & Programme5 \\
\cline { 2 - 11 } & t/min & Number & t/min & Number & t/min & Number & t/min & Number & t/min Number \\
\hline A & 110 & 3 & 90 & 4 & 75 & 5 & 60 & 6 & \\
B & 180 & 2 & 90 & 4 & 60 & 6 & & & \\
C & 360 & 2 & 270 & 3 & 210 & 4 & & & \\
D & 180 & 2 & 90 & 4 & 60 & 6 & & & & \\
E & 585 & 2 & 450 & 3 & 375 & 4 & 330 & 5 & 270 & 6 \\
F & 375 & 2 & 280 & 3 & 190 & 4 & & & & \\
G & 330 & 1 & 190 & 2 & & & & & & \\
H & 130 & 1 & 60 & 2 & 50 & 3 & 40 & 4 & & \\
I & 1600 & 1 & 930 & 2 & 720 & 3 & 465 & 4 & 340 & 5 \\
J & 990 & 2 & 750 & 3 & 520 & 4 & 425 & 5 & 350 & 6 \\
K & 90 & 2 & 75 & 3 & 65 & 4 & & & & \\
L & 180 & 2 & 140 & 3 & 90 & 4 & & & \\
M & 280 & 2 & 235 & 3 & 160 & 4 & 125 & 5 & 80 & 6 \\
N & 900 & 2 & 680 & 3 & 480 & 4 & 390 & 5 & 320 & 6 \\
\hline
\end{tabular}




\begin{tabular}{ccccccccccc}
\hline $\mathrm{O}$ & 630 & 2 & 475 & 3 & 280 & 4 & 210 & 5 & 145 & 6 \\
$\mathrm{P}$ & 1500 & 1 & 810 & 2 & 670 & 3 & 545 & 4 & & \\
$\mathrm{Q}$ & 270 & 2 & 220 & 3 & 145 & 4 & 120 & 5 & 75 & 6 \\
$\mathrm{R}$ & 750 & 1 & 360 & 2 & 290 & 3 & & & & \\
$\mathrm{~S}$ & 150 & 3 & 90 & 4 & 75 & 5 & 65 & 6 & & \\
$\mathrm{~T}$ & 245 & 3 & 210 & 4 & 185 & 5 & 160 & 6 & & \\
$\mathrm{U}$ & 370 & 1 & 180 & 2 & 145 & 3 & & & & \\
$\mathrm{~V}$ & 390 & 1 & 180 & 2 & & & & & & \\
$\mathrm{~W}$ & 1635 & 2 & 1450 & 3 & 950 & 4 & 800 & 5 & 680 & 6 \\
$\mathrm{X}$ & 135 & 2 & 90 & 3 & 64 & 4 & 50 & 5 & & \\
$\mathrm{Y}$ & 1300 & 3 & 1150 & 4 & 920 & 5 & 780 & 6 & & \\
\hline
\end{tabular}

When search the optimal solution using Ant Colony Algorithm, in order to ensure the solution quality, the parameters are set as follow. The maximum cyclic number $N_{\max }=200$, the ant number $M=20$, the pheromone intensity $Q=100$. The value of $\alpha, \beta$ and $\rho$ using the dynamic adjustment method, when the cycle numbers are less than 50 times, $\alpha=0.5, \beta=1$ and $\rho=0.3$, in the next cycle, the parameters are adjusted to $\alpha=1, \beta=5$ and $\rho=0.1$. According to the basic process and algorithm flow chart of the paper, the results of cycle 10 times are analyzed. In order to facilitate statistics, the task of the project is converted to hours (not reserved) in the calculation results[11]. The results are shown in Table 2.

Table 2 Analysis of results of experiments

\begin{tabular}{ccccc}
\hline $\begin{array}{c}\text { Calculatio } \\
\text { n Times }\end{array}$ & $\begin{array}{c}\text { Task } \\
\text { Duration/h }\end{array}$ & Number & $\begin{array}{c}\text { First } \\
\text { Search } \\
\text { Iteration } \\
\text { Number }\end{array}$ & $\begin{array}{c}\text { Computin } \\
\text { g Time/s }\end{array}$ \\
\hline 1 & 134 & 6 & 117 & 6.93 \\
2 & 138 & 6 & 128 & 7.5 \\
3 & 134 & 6 & 123 & 7.32 \\
4 & 123 & 7 & 119 & 6.97 \\
5 & 134 & 6 & 104 & 5.89 \\
6 & 138 & 6 & 109 & 6.02 \\
7 & 134 & 6 & 129 & 7.71 \\
8 & 142 & 6 & 116 & 6.85 \\
9 & 134 & 6 & 122 & 7.27 \\
10 & 134 & 6 & 115 & 6.85 \\
\hline
\end{tabular}

\subsection{Result analysis}

The calculation results show that the optimal scheme with the number of personnel for 6 , and the maintenance period is about 15 18 days (8 hours a day). According to the algebra and time of the first search, the calculation results are stable. Since the method is of great convergence, the better optimal solution can be obtained.

In the condition that the maintenance time and the latest start time are satisfied, the total number of maintenance personnel can be compared, if the maximum maintenance time is the same. The program with less personnel can improve the support ability of maintenance support unit, and the maintenance team can be quickly put into a new task.

There are many different kinds of optimal solutions for various objectives, which meet the constraint condition. It is necessary for obtaining the optimal maintenance scheme with the actual situation to expand the scope of the target optimization solution, When considering the comprehensive optimization of multiple targets.

Under the condition of meeting the time limit, the actual conditions should be fully considered, the actual constraints of the maintenance and repair should be increased, so that the optimal solution can 
be more accurately find. In this example, if the number of maintenance personnel and maintenance costs is enough, the maintenance personnel should be more sent to, so that the task can be completed quickly. So, the constraints of the number of maintenance personnel should be added, the optimal solution can be found in the number of more person.

\section{Conclusions}

In the process of building maintenance support force, the core problem is how to determine the number of basic maintenance units according to maintenance tasks, types of maintenance personnel and number of personnel. Under this current situation, the maintenance support unit is systematically analysed, and a multi-objective optimization model is established based on time constraints and personnel. The specific methods and steps are given as well. It provides technical support for the establishment of the maintenance support unit, and enriches the theory of maintenance support force construction.

The optimal maintenance personnel combination can be obtained through the research. a method was established to determine the basic unit of maintenance support personnel based on the maintenance tasks, personnel requirement and personnel characteristics. The method also provides a basis for the rational use of maintenance resources. On the other hand, according to the change of maintenance task scale, quantitative analysis for all kinds of personnel can be obtained.

re has multiple execution plans. Each one is composed of a fixed repair time and a fixed number of maintenance personnel. In order to minimize the total duration and total number of people at the same time, it is the problem of multi objective optimization.

\section{References}

[1]"the battle equipment" Science Review Committee. The Battle Equipment [M]. Beijing: National Defense University press, 2002:16- 33 (in Chinese)

[2] Lei Deming, Yan Xinping. Multi Target Intelligent Optimization Algorithm and Its Application [M]. Beijing: Science Press, 2009:78- 85 (in Chinese)

[3]Huang Ping, Meng Yonggang. Optimization Theory and Method [M]. Beijing: Tsinghua University press, 2009:35- 45 (in Chinese)

[4]Li Wanqing, Meng Wenqing. Engineering Network Planning Technology [M]. Beijing: Science Press, 2009:127- 150 (in Chinese)

[5]Daisy X S, Thomas N, Mohan M K. Applying a Genetic Algorithm-Based Multi Objective Approach for Time-Cost Optimization[J]. Journal of Construction Engineering and Management, 2004,130 (2): 168-176.

[6]Chen Baowen. Research on the Application of Ant Colony Optimization Algorithm in Vehicle Routing Problem [D]. Harbin: Harbin Institute of Technology, 2009 (in Chinese) [7]Wang Tao, Zhang Fulong, Li Guangfeng. Optimization of Maintenance Support Resource Scheduling Based on Multi-Objective Genetic Algorithm ([J].), 2013, 21 (11): 3017-3020 (in Chinese)

[8]Chen Changmin, Xie Weicheng, Fan Songsong. Adaptive and Max Min Ant Colony Algorithm of Logistics Vehicle Routing Optimization [J]. Journal of Xihua University, 2011, 30 (3):5-8 (in Chinese)

[9]Zhang Yuhua, Pan Yu. Research on Emergency Logistics Distribution Vehicle Scheduling Based on Ant Colony Algorithm [J]. logistics technology, 2009, 32 (5):47-49 (in Chinese)

[10]Tang Lianggui, Cheng Daijie. Research on Logistics Scheduling Algorithm [J]. based on Ant Colony Optimization in Computer Engineering, 2006, 32 (7):177-179 (in Chinese)

[11]Duan Haibin. Principle of Ant Colony Algorithm and Its Application [M]. Beijing: Science Press, 2005:224- 260 (in Chinese) 